\title{
Percepçôes de obesos deprimidos sobre os fatores envolvidos na manutenção da sua obesidade: investigação numa unidade do Programa Saúde da Família no município do Rio de Janeiro
}

\section{I ${ }^{1}$ Angela Lessa Moraes, ${ }^{2}$ Eliane Carnot Almeida, ${ }^{3}$ Luciana Borges Souza |}

Resumo: Obesidade e depressão, segundo a OMS, estão entre os maiores problemas de Saúde Pública no mundo. Diversos estudos apontam para importante associação entre ambas, o que pode resultar no agravamento do prognóstico das comorbidades, menor aderência ao tratamento, maior morbimortalidade, assim como comprometimento funcional e psicossocial dos obesos. Este artigo resulta de pesquisa para dissertação de mestrado em Saúde da Família, cujo objetivo foi investigar a percepção de obesos com depressão sobre os fatores envolvidos na manutenção da própria obesidade. Estudo exploratório, qualitativo, realizado em uma unidade de Saúde da Família no Rio de Janeiro. Foram selecionados aleatoriamente 68 usuários obesos, sendo 21 identificados com escore para depressão a partir da aplicação do Inventário Beck de Depressão. Destes, 19 foram entrevistados individualmente e os dados obtidos foram trabalhados a partir da técnica de análise de conteúdo. Destacaram-se, como categorias principais, o sofrimento físico, o sedentarismo, o sofrimento emocional, a insatisfação com a autoimagem, a alimentação inadequada, o convívio familiar, social e a motivação para mudanças. Agrupadas, essas categorias evidenciaram cinco eixos temáticos: a percepção da obesidade, o comportamento alimentar, o ambiente familiar, o retraimento social e a falta de motivação. A análise das percepções dos entrevistados permitiu mapear uma teia complexa de fatores mutuamente reforçadores envolvidos com a manutenção da obesidade, que são agravados quando esta é associada à depressão. Os resultados da pesquisa podem contribuir para subsidiar estratégias de atenção integral e diferenciada à saúde dos obesos, sobretudo os que apresentam depressão.

\author{
1 Médica psiquiatra; mestre \\ em Saúde da Família pela \\ Universidade Estácio de \\ Sá. Endereço eletrônico: \\ angelamo52@gmail.com \\ 2 Psicóloga; doutora em \\ Saúde Coletiva pelo Instituto \\ de Medicina Social da \\ UERJ; professora titular da \\ Universidade Estácio de \\ Sá; professora adjunta da \\ Universidade Federal do Estado \\ do Rio de Janeiro (UNIRIO). \\ Endereço eletrônico: ecarnot@ \\ uol.com.br \\ ${ }^{3}$ Médica pediatra; doutora \\ em Saúde da Criança e da \\ Mulher pelo Instituto Fernandes \\ Figueira/FIOCRUZ; professora \\ do Curso de Medicina do \\ Centro Universitário Serra dos \\ Órgãos e do Curso de Medicina \\ e do Mestrado em Saúde da \\ Família da Universidade Estácio \\ de Sá. Endereço eletrônico: \\ luborges10@gmail.com
}




\section{Introdução}

A obesidade afeta cerca de 500 milhões de pessoas em todo o mundo (WHO, 2012) e, no Brasil, atinge $15,8 \%$ da população adulta (IBGE, 2010), demandando ações estratégicas como prioridades do Sistema Único de Saúde SUS (MALTA et al., 2006).

Considerada um problema de saúde pública (WHO, 2012), a obesidade é um distúrbio crônico de etiologia multifatorial e correlação com outras doenças crônicas que diminuem a duração e a qualidade de vida do indivíduo, sendo responsáveis por elevado ônus social e econômico (COUTINHO; GENTIL; TORAL, 2008). É definida como acúmulo excessivo de tecido adiposo no organismo, em nível que pode comprometer a saúde, sendo o indicador preconizado para sua avaliação o índice de massa corporal $\left(\mathrm{IMC}=\right.$ peso/altura $\left.{ }^{2}\right)$ igual ou superior a $30 \mathrm{~kg} / \mathrm{m}^{2}$ (WHO, 1998). A combinação do IMC com outros indicadores, como a presença de dobras cutâneas e a localização da gordura, indicada pela relação cintura-quadril (RCQ $>1$, homens; $\mathrm{RCQ}>0,85$, mulheres) e pela circunferência da cintura $(\mathrm{CC}>95 \mathrm{~cm}$, homens; CC>80 cm, mulheres), permite um melhor diagnóstico da obesidade, incluindo os fatores de risco para doenças crônicas (BRASIL, 2006).

A depressão, também considerada um problema de saúde pública (WHO, 2011), afeta aproximadamente 10 milhões de pessoas no Brasil (BRASIL, 2012) e tem prevalência em obesos de $25 \%$ (ABESO, 2011) a 30\% (SIMON et al., 2008). Por ser distúrbio crônico e recorrente, é responsável por incapacitação laboral e ônus social (FLECK, 2009) e, quando associada à obesidade, é de difícil diagnóstico e tratamento (ONYIKE et al., 2003).

Segundo o Manual Diagnóstico e Estatístico de Transtornos Mentais - DSM IV-TR (APA, 2002), os sintomas clínicos da depressão incluem: humor triste, perda de interesse e prazer, distorções do apetite, alterações do peso, sentimento de inutilidade, culpa excessiva, baixa autoestima, fadiga, perda de energia e de motivação. A falta de motivação, em particular, pode implicar fraca adesão ao tratamento da obesidade, quando preconizado (SOARES, 2008). Deve-se ressaltar que o sofrimento psíquico classificado como depressão revela aspectos complexos da subjetividade e das condiçōes sociais dos indivíduos. Esse sofrimento, que pode incluir tristeza, apatia, busca da identidade etc., representa enormes desafios para a clínica psiquiátrica (BARBOSA, 2006). Além disso, o 
critério diagnóstico da depressão, segundo o DSM IV, não impede a inclusão de manifestações subjetivas associadas a situações comuns de vida, levando a um grande número de falsos positivos (FREITAS, 2011; CHALUB, 2010).

Embora alguns autores apontem a obesidade como causadora e não como consequência de sofrimento psíquico (OLIVEIRA; LINARDI; AZEVEDO, 2004), outros afirmam que indivíduos com depressão apresentam maior risco para a obesidade (BRUCE, 2008). A Associação Brasileira para o Estudo da Obesidade e da Síndrome Metabólica (ABESO, 2009) destaca que a obesidade aumenta em $55 \%$ o risco de depressão, e esta, em 58\% o risco de obesidade, indicando possível circularidade entre as duas patologias. Ball e Atlantis (2008) indicam que, mais do que a obesidade em si mesma, a percepção de estar acima do peso é fator de risco para sintomas depressivos. Além disso, fatores psicossociais, como estigma e discriminação, também podem contribuir para a depressão em obesos (SEGAL; CARDEAL; CORDÁS, 2002).

Segundo Goffman (1988), estigma é a identidade atribuída pela sociedade àqueles que não respondem aos padrões e características estabelecidos, o que os torna diferentes. No caso dos obesos, o caráter negativo do estigma se intensifica na medida em que as expectativas e exigências sociais os tornam indesejados, depreciados e marginalizados (MATTOS; LUZ, 2009). Ou seja, obesidade não é apenas um problema individual, é também social.

Como exemplos de atitudes e estereótipos negativos em relação à obesidade desde a infância, Staffiere (1967) relata como crianças descreviam outras, desenhadas como obesas: preguiçosas, burras e mentirosas. Segal (2000) ressalta que os obesos permanecem menos tempo na escola e posteriormente têm menos chances em empregos mais concorridos ou têm salários mais baixos. Alguns obesos, segundo o autor, têm também a percepção de serem tratados desrespeitosamente por profissionais de saúde, o que os leva a relutar em procurar ajuda relacionada a sua condição. Os padrões ideais de beleza da sociedade também elaboram mensagens que definem uma identidade negativa e depreciada dos obesos, promovendo um sentimento de desqualificação que prejudica sua inserção social e gera sofrimento subjetivo (FELIPPE, 2001).

Reforçando esse quadro, à percepção negativa do corpo obeso somamse outras, igualmente negativas, dos deprimidos, tais como sentimentos de insuficiência, incapacidade, vergonha e autodepreciação, contribuindo na 
manutenção da obesidade (UEHARA; MARIOSA, 2005). Tornando o desafio ainda mais preocupante, a depressão, na maioria dos casos, não é adequadamente diagnosticada, principalmente quando se expressa por sinais e sintomas físicos (WHO, 2011). A mudança de hábitos é indispensável para o enfrentamento da obesidade, para o que a motivação é primordial e especialmente difícil de ser alcançada nos deprimidos (BERKEL et al., 2005).

O presente artigo, elaborado a partir de pesquisa realizada para o mestrado em Saúde da Família, teve como objetivo investigar as percepções de obesos deprimidos sobre os fatores envolvidos na manutenção da sua obesidade, visando a um entendimento mais amplo dos desafios para o enfrentamento desta, especialmente quando agravada pela depressão. Para isso, abordamos os fatores psicossociais e o entendimento psicodinâmico de como o indivíduo que apresenta obesidade e depressão organiza seu mundo interior, a percepção dos próprios sentimentos, seus mecanismos de defesa e adaptação, seu autoconceito, sua autoimagem e sua motivação para mudanças. Considerando que o ato de comer tem aspectos eminentemente sociais e culturais, buscou-se estudar também a relação deste com as emoções, estilo de vida e experiências familiares e sociais. Finalmente, entendendo que a obesidade não pode ser enfrentada sem uma participação ativa, consciente e permanente dos próprios sujeitos obesos, é essencial conhecer como eles percebem e o que pensam sobre seu problema (PINTO; BOSI, 2010).

Apesar de a obesidade ser reconhecida como evento de controle prioritário (PINHEIRO; FREITAS; CORSO, 2004), o que se observa é que poucas são as ações que tratam o obeso na sua integralidade (GREJANIN et al., 2007). As diretrizes para o atendimento ao obeso do Ministério da Saúde (BRASIL, 2006), por exemplo, priorizam o modelo biológico, com orientação nutricional, atividades físicas e tratamento clínico das comorbidades. No entanto, como anteriormente discutido, fatores psíquicos e sociais podem agravar ou serem agravados pela obesidade, sobretudo quando associada à depressão (DOBROW; KAMENETZ; DEVLIN, 2002).

\section{Método}

Esta pesquisa foi realizada na unidade Lapa, no município do Rio de Janeiro, do Programa de Saúde da Família (PSF). O PSF constitui um ambiente propício para a atenção aos problemas das doenças crônicas, incluindo a obesidade e 
suas comorbidades. Trata-se de estudo exploratório, de natureza qualitativa, fundamentado na apreensão das atitudes, crenças e motivações (MINAYO, 2004).

A seleção dos sujeitos baseou-se nos prontuários dos obesos cadastrados na unidade. Foram definidos como critérios de inclusão: adultos (20 a 59 anos), residentes com a família, com a escolaridade igual ou superior ao $1^{\circ}$ grau completo; e, como critérios de exclusão, aqueles com doenças endócrinas ou psicóticas e gestantes. A amostra, por seleção aleatória, indicou 68 sujeitos, aos quais foi apresentado o objetivo da pesquisa e feito o convite para participar da mesma. Todos assinaram o Termo de Consentimento Livre e Esclarecido.

Para identificação dos sujeitos deprimidos, utilizou-se o Inventário Beck de Depressão, instrumento validado no Brasil e amplamente utilizado em pesquisas (CUNHA, 2001). Foram identificados 21 sujeitos com escore para depressão e, destes, 19 aceitaram ser entrevistados individualmente, com roteiro semiestruturado, tendo cada entrevista a duração média de 45 minutos, entre junho e agosto de 2011. Todas as entrevistas foram gravadas e transcritas pela pesquisadora. Nenhum dos sujeitos estava em tratamento para depressão ou tinha sido diagnosticado anteriormente como deprimido. Todos foram orientados pela pesquisadora a procurar a unidade para atendimento psicológico e/ou psiquiátrico.

$\mathrm{O}$ roteiro das entrevistas abordou os seguintes aspectos: sentimento em relação à própria obesidade, hábitos alimentares, atividade física regular, ambiente familiar, interação social e motivação para mudanças. $\mathrm{O}$ tratamento e a interpretação dos dados basearam-se na técnica da análise de conteúdo, sendo agrupados em categorias temáticas (BARDIN, 2004). Na análise destacaram-se, como categorias principais do discurso dos entrevistados, os fatores percebidos por eles como associados à manutenção da sua obesidade, tais como: sofrimento físico, sedentarismo, sofrimento emocional, insatisfação com a autoimagem, comportamento alimentar inadequado, ambiente familiar e social, assim como falta de motivação para mudanças de hábitos.

Agrupadas, essas categorias evidenciaram cinco eixos temáticos: 1) a percepção da própria obesidade; 2) o comportamento alimentar; 3) o ambiente familiar; 4) o retraimento social; e 5) a falta de motivação. Buscou-se relacionar a percepção dos fatores envolvidos na manutenção da obesidade à experiência dos obesos deprimidos na materialidade de suas vidas, seguindo o objetivo da pesquisa. Ao final, buscou-se reunir as diferentes percepções num mapa conceitual único, 
mostrando as múltiplas e complexas interações possíveis entre os diferentes fatores mantenedores da obesidade, tal como percebidos pelos sujeitos desta pesquisa.

O estudo teve aprovação do Comitê de Ética em Pesquisa da Universidade Estácio de Sá (Protocolo: 00230308000-11) e seguiu as recomendaçōes da Resolução no 196/96, que normatiza as pesquisas envolvendo seres humanos (BRASIL, 1996). Para manter o sigilo, os fragmentos de depoimentos transcritos foram identificados pela letra E (Entrevistado) seguida de um número arábico.

\section{Resultados e discussão}

Participaram da pesquisa 45 mulheres e 23 homens. O Inventário Beck de Depressão indicou 31\% destes (seis homens e 15 mulheres) com escore para depressão, índice compatível com os da literatura (ABESO, 2011; SIMON et al., 2008). As percepções dos obesos deprimidos sobre os fatores envolvidos na manutenção da sua obesidade são apresentadas e analisadas a seguir, associadas a cada um dos cinco eixos temáticos citados no Método.

Deve-se ressaltar que os entrevistados, em seus relatos, percebem com frequência relacionamentos causais entre esses eixos, ou seja, eles não são isolados.

\section{A percepção da própria obesidade}

Este eixo evidenciou o agrupamento das categorias "sofrimento físico", "sedentarismo", "sofrimento emocional" e "insatisfação com a autoimagem".

\section{Sofrimento físico}

O sofrimento físico que acompanha o obeso, como dor e prejuízo na movimentação, pode ser entendido como causa ou consequência da obesidade (DÂMASO, 2003).

Em alguns depoimentos, a percepção da dor é relatada como limitante, relacionando-se com a dificuldade no manejo do próprio corpo e com o sentimento de perda de autonomia, o que é especialmente preocupante, visto que "a autonomia implica na construção da capacidade de corresponsabilização pelo cuidado consigo, com os outros e com a vida" (CAMPOS; BARROS; CASTRO, 2004, p. 745):

Tenho problemas de coluna, fadiga, dificuldade de me calçar, não dá para abaixar, não consigo me lavar direito e sinto muita dor, sempre (E8). 
Não consigo fazer certos movimentos, dói tudo. É triste não poder cuidar da casa,

pensar em subir num ônibus e o corpo não corresponder (E14).

Pode-se inferir, pelos relatos acima, o quão desafiador pode ser, para esses sujeitos, realizar os exercícios físicos regulares recomendados como importantes coadjuvantes no enfrentamento da obesidade (BRASIL, 2006), e como a percepção da própria obesidade se relaciona ao sentimento de limitação, reforçando o sedentarismo, o que não contribui para a redução do peso.

A dor também parece, nesses depoimentos, estar associada à tristeza, ao sentimento de incapacidade, de inutilidade e à autodepreciação, sintomas presentes na depressão. Fortes (2002, p. 343) ressalta que as características cognitivas da depressão e da dor crônica são semelhantes, e que "a relação entre dor e depressão é frequente e intensa, na qual dor gera depressão e depressão gera dor". Isso torna bastante desafiador o diagnóstico de depressão nos pacientes com dor crônica.

\section{Sofrimento emocional}

Araújo (2004) destaca o sofrimento emocional como fator retroalimentador na manutenção da obesidade. Os depoimentos indicam que a dificuldade para lidar com emoções negativas estimula a busca do alimento como conforto:

Quando fico nervosa, como o dobro. Como para ficar mais relaxada (E10).

Fiquei obeso devido a problemas. Sabe como é, comer para esquecer? Meus pais brigavam, eu ficava nervoso e comia (E19).

Por outro lado, conflitos relacionados à culpa pelo consumo calórico, ao ganho de peso e às doenças promovem baixa autoestima e maior consumo calórico, num círculo vicioso (MARCHESINI, 2007). O sentimento de culpa, reforçando o consumo alimentar, se revela nos depoimentos:

O que me traz remorso é a diabete. Não me cuidei, não segui o protocolo para perder peso. Aí vem o remorso que não traz reação [...], como mais ainda (E1).

Não estou gostando de mim gorda assim, choro o tempo todo e aí como mais (E8).

É uma tristeza, engordei desesperadamente, aí eu como para aliviar esse sofrimento (E10).

Como observado acima, alguns percebem o caráter de circularidade e autorreforço dos fatores associados à manutenção da sua obesidade. 


\section{Insatisfação com a autoimagem}

A percepção da própria obesidade, em alguns casos, é deflagrada pela insatisfação com a autoimagem e se expressou, em alguns depoimentos, como desqualificação e desvalia de si, corroborando o que Stunkard e Wadden (1992) denominaram de "evitação do espelho":

No dia que vi meu corpo inteiro no espelho, eu me assustei! Estou gorda assim? [...] não gosto de me olhar no espelho [...] é só para me pentear (E4).

Felippe (2001) fala também do sentimento de humilhação, associado, entre outras coisas, à dificuldade de o obeso encontrar um manequim que lhe sirva:

É muito difícil encontrar roupa que me serve [...] volto mais deprimida ainda. Igual agora, no inverno, os casacos não fecham (E3).

As roupas ficam apertadas, me sinto triste. Só uso cor escura (E9).

Aqui não fica explícita a percepção da causalidade "insatisfação $\rightarrow$ obesidade" (comer mais por consolo), mas a "obesidade $\rightarrow$ "insatisfação" $\rightarrow$ "depressão". Por outro lado, a percepção negativa do corpo obeso é acompanhada, nos indivíduos com depressão, por sentimentos de autorrecriminação e autodepreciação (DIXON; DIXON; O’BRIEN, 2003), o que promove mais isolamento social.

Não estou gostando de mim [...] muito gorda, choro o tempo todo, é minha culpa, acho que estou incomodando as pessoas, por isso fico em casa sozinha (E12).

Ainda com relação à insatisfação com a autoimagem, os homens enfocaram as limitações físicas, o que é compatível com a construção social dos papéis masculino e feminino desde a infância (BENEDETTO, 2004). Estimula-se nos meninos o desenvolvimento da força; também são educados para suportar sem chorar suas dores físicas e emocionais, reforçando as dificuldades para verbalizar as próprias necessidades (BRAZ, 2005). Além disso, os homens desconsideram a obesidade como um problema, a não ser quando traz sinais e sintomas de desconforto e de incapacidade física (BRASIL, 2006).

O que incomoda? A barriga. A gente quer abaixar e não pode. [...] até para calçar um sapato [...] a barriga cria uma barreira física até na hora da relação (E 18).

Essas particularidades masculinas podem indicar a necessidade de diferentes abordagens de tratamento para os homens. Figueiredo (2004), por exemplo, propóe estratégias e atividades especificamente dirigidas à subjetividade masculina, tais como grupos de discussão para homens ou mesmo atendimento individual, em 
espaços diversos (PSF ou comunidade) e em horários mais flexíveis, compatíveis com os do trabalho, possibilitando maior participação.

\section{O comportamento alimentar}

A percepção sobre o comportamento alimentar que emergiu das entrevistas também sugere uma complexa relação com o sofrimento emocional, com o estilo de vida e com as experiências familiares e relaçôes sociais (MATOS, 2006).

Do ponto de vista nutricional, a ingestão calórica de alguns obesos é descrita como nociva e inadequada, decorrência tanto da grande quantidade consumida quanto da dieta de maior densidade energética, estimulada pela industrialização dos alimentos, ou a combinação dos dois (ALVARENGA, 2004). Por isso, no campo da atenção primária aos obesos, o nutricionista atua com prescrições dietoterápicas e práticas educativas em nutrição. Porém, ele deve considerar, sempre, os fatores psíquicos que podem influenciar o comportamento alimentar, como o contexto familiar e o social, indicando, no trabalho integrado com outros profissionais, situações de risco associadas à obesidade, tais como desemprego, baixa autoestima e outros problemas emocionais (BRASIL, 2006). Além disso, Araújo (2004, p. 114) ressalta que, do ponto de vista psicológico, o comportamento alimentar é um processo relacional carregado de significado emocional, e que "emocionalmente a conduta alimentar inadequada tem uma função: proteger o obeso de sofrimentos psíquicos que ele imagina serem insuportáveis”.

A ingestão calórica excessiva pode servir tanto como gratificação substituta, ou seja, um equivalente do afeto, quanto um mecanismo de defesa para aplacar angústias, tensões emocionais, e pode ter como fatores precipitantes dificuldades para lidar com situações negativas da vida (MATOS, 2006).

Perdi trabalho, marido e aí comecei a engordar. Substituía as coisas por comida [...] percebi isso no dia de pagar as contas; comecei a comer e não parei (E4).

No entanto, o comportamento alimentar nem sempre tem função protetora. Pode ser um sintoma manifesto de transtorno alimentar quando não se consegue parar de comer. É o caso, por exemplo, da compulsão alimentar, caracterizada pela ingestão, num curto intervalo de tempo, de quantidade de alimento maior do que a maioria das pessoas consumiria e pelo sentimento de perda de controle sobre esse episódio (SEGAL; CARDEAL; CORDÁS, 2002). 
Outro dia, comi feijāo, arroz, carne e brigadeiro, tudo junto tentando me acalmar, acredita? (E11).

O consumo alimentar excessivo também foi relacionado à autoavaliação negativa pela falta de autocontrole e autoaceitação, concordando com Cash e Deagle (1997).

Sou feia, gorda e sem controle, como muito e às vezes nem estou com fome (E3).

Me sinto uma fracassada. Sei tudo de dieta, mas não faço. É como se fosse uma viciada em drogas (E13).

Como muita besteira, acho que tenho raiva de mim e não me controlo (E16).

Dois aspectos se destacam nesses relatos: um é que o hábito alimentar é percebido como inadequado, podendo estar associado a descontrole; o outro é que a "causa" desse hábito seria um sofrimento emocional, com a alimentação servindo de compensação. Nesses depoimentos, a percepção da circularidade dos fatores causais associados à manutenção da obesidade está presente, de várias formas, como, por exemplo: "obesidade $\rightarrow$ autodepreciação $\rightarrow$ consumo alimentar $\rightarrow$ obesidade $\rightarrow$ autodepreciação $\rightarrow$ autossabotagem $\rightarrow$ consumo alimentar $\rightarrow$ obesidade".

\section{$\mathrm{O}$ ambiente familiar}

A família é organizadora dos hábitos de vida e alimentares, atuando como modelo nos vínculos atuais e transgeracionais (SANTOS; RABINOVICH, 2011). Quando toda a família é de obesos, o desajuste alimentar é mantido como forma de autodefinição e lealdade ou, inversamente, como consequência da rebeldia contra as tentativas de controle pela própria família, levando a pessoa a comer mais ainda (BENEDETTI, 2006). Os relatos confirmaram a percepção dessa influência familiar como justificativa para a manutenção da obesidade:

Toda a minha família é gordinha, dizem que a gente tem que comer pra ficar forte, eles acham que gente magra é feia. Se depender deles, eu não emagreço (E6).

A minha família é obesa. Temos o mesmo biotipo. É normal para nós ser obesos; quando falo de dieta, é a maior "zoação", e acabo voltando aos maus hábitos (E14).

A família também pode contribuir para a disseminação do estigma e da baixa autoestima em obesos, com recriminações, comentários e apelidos depreciativos. Tais atitudes podem ser sentidas como exclusão afetiva, reforçada pela cobrança de caráter altamente depreciativo e desmotivador (BURD, 2004). 
Minha mulher me apelidou de Gordo e Balofo [...] me sinto mal, em casa não recebo apoio, só apelidos (E15).

Marido diz: "Está uma baleia, hein?”, fico magoada e perco a motivação (E11).

O meu pai fala: tem que andar, tem que emagrecer. Eu me sinto pressionada e a cobrança desestimula [...] dá vontade de comer mais (E18).

\section{$\mathrm{O}$ retraimento social}

O quarto eixo temático evidenciado nas entrevistas diz respeito ao retraimento social relacionado à vivência da obesidade. Observou-se que a percepção negativa do corpo obeso se soma a outras percepções igualmente negativas que acompanham os deprimidos, tais como autodepreciação e vergonha (UEHARA; MARIOSA, 2005), que reforçam a dificuldade de interação social:

O ambiente de academia não é para mim [...] me senti deslocada, todo mundo saradão e eu horrorosa, não fui mais [...] praia, só quando não tem ninguém (E4).

Eu tenho uma vergonha terrível de ser gordo. No trabalho me botaram o apelido de baleia. [...] acho até que me mandaram embora por causa da gordura (E18).

Para Pinto e Bosi (2010), o estigma da obesidade, quando presente, reforça o sentimento de depreciação, desvalorização, constrangimento e vergonha, influenciando as relações sociais, ocupacionais e o autoconceito, podendo levar o indivíduo ao isolamento e ao retraimento social.

"Você voltou a engordar de novo. Vai ficar entalada na roleta!". Não gosto de ser criticada. Prefiro nem sair de casa (E12).

Ficam encarnando em mim. O preconceito com a gordura não é de forma explícita e sim em forma de piadas, eu percebo os comentários, aí fico em casa (E14).

\section{A falta de motivação}

Este eixo temático engloba alguns fatores que interferem no relacionamento afetivo, social e ocupacional dos entrevistados e que podem estar associados à presença de depressão, como ressalta Fleck (2009).

Desanimado, não tenho disposição para fazer as coisas em casa, para nada, até na relação sexual, só faço porque tenho que fazer (E10).

$\mathrm{O}$ obeso deprimido tem pouco interesse e energia para mudanças, além de apresentar percepção negativa de si (BUSSE, 2007). Ora, essas mudanças de hábitos de vida, indispensáveis para o sucesso do tratamento da obesidade, 
demandam, entre outras coisas, um forte componente motivacional condicionado por disposição interna (BERKEL et al., 2005), algo difícil de alcançar nesse quadro. Como a intensidade da motivação é determinada não só pelo desejo da realização, mas também pela probabilidade de sucesso (KAPLAN; SADOCK, 2007), a frustração pela demora em obter resultados satisfatórios dos esforços para emagrecer foi relatada pelos entrevistados como fator significativo de desmotivação:

Você fica a vida toda fazendo dieta sem solução e chega a um ponto que cansa [...] não comia quase nada [...] fiquei sem força para continuar, aí desisti (E3).

Doze entrevistados relataram algum tipo de insatisfação, desmotivação e falta de ânimo para mudar sua condição de obeso, apesar de saberem que essa condição promove comprometimentos físicos e emocionais que pioram sua qualidade de vida.

A depressão me deixa com uma lentidão maior e com o controle remoto na mão, não tenho vontade de sair e fazer coisas. Se pudesse, só ficava em casa (E1).

Sem disposição para acordar. De manhã é o pior horário. Sei que a obesidade dá problemas. Tinha disposição para caminhar apesar do peso, não tenho mais (E14).

Se pudesse, ficava quietinho no meu quarto, trancado e sempre me sentindo cansado para tudo. A obesidade me deixa tímido, a tristeza é de outro lugar (E19).

Embora a percepção de cada sujeito sobre os fatores que reforçam ou mantêm sua obesidade varie em ênfase e grau de consciência, este estudo indica a existência de complexas interações, não só entre aqueles fatores, mas também, globalmente, entre os diferentes eixos temáticos. Ou seja, na avaliação ou tratamento de um paciente obeso com depressão, é possível que estejam presentes, em cadeia, uma ou mais interações e efeitos de reforço e feedback mantenedores da obesidade, demandando uma atenção muito cuidadosa por parte dos profissionais de saúde responsáveis pelo tratamento. Isso inclui escutar e valorizar as particularidades de cada caso, estabelecendo uma relação humanizada e contextualizada com a família e a rede social, identificando as dimensôes subjetivas e as determinações psicossociais envolvidas nessas formas de adoecer (BRASIL, 2006).

Mais especificamente, a questão das circularidades da obesidade em geral, ressaltada por Hamid (2009), aparece, quando ela está associada à depressão, mais claramente e com maior abrangência ainda pela importância dessas dimensões psicossociais. Essa teia sistêmica de interações possíveis está representada na 
figura 1. Nela, os círculos maiores representam os eixos temáticos; os retângulos

parcialmente encobertos por esses círculos, os fatores causais percebidos (categorias) associados a cada um; e as flechas, as diferentes interaçōes de reforço.

\section{Figura 1 (Adaptada de MORAES, 2011)}

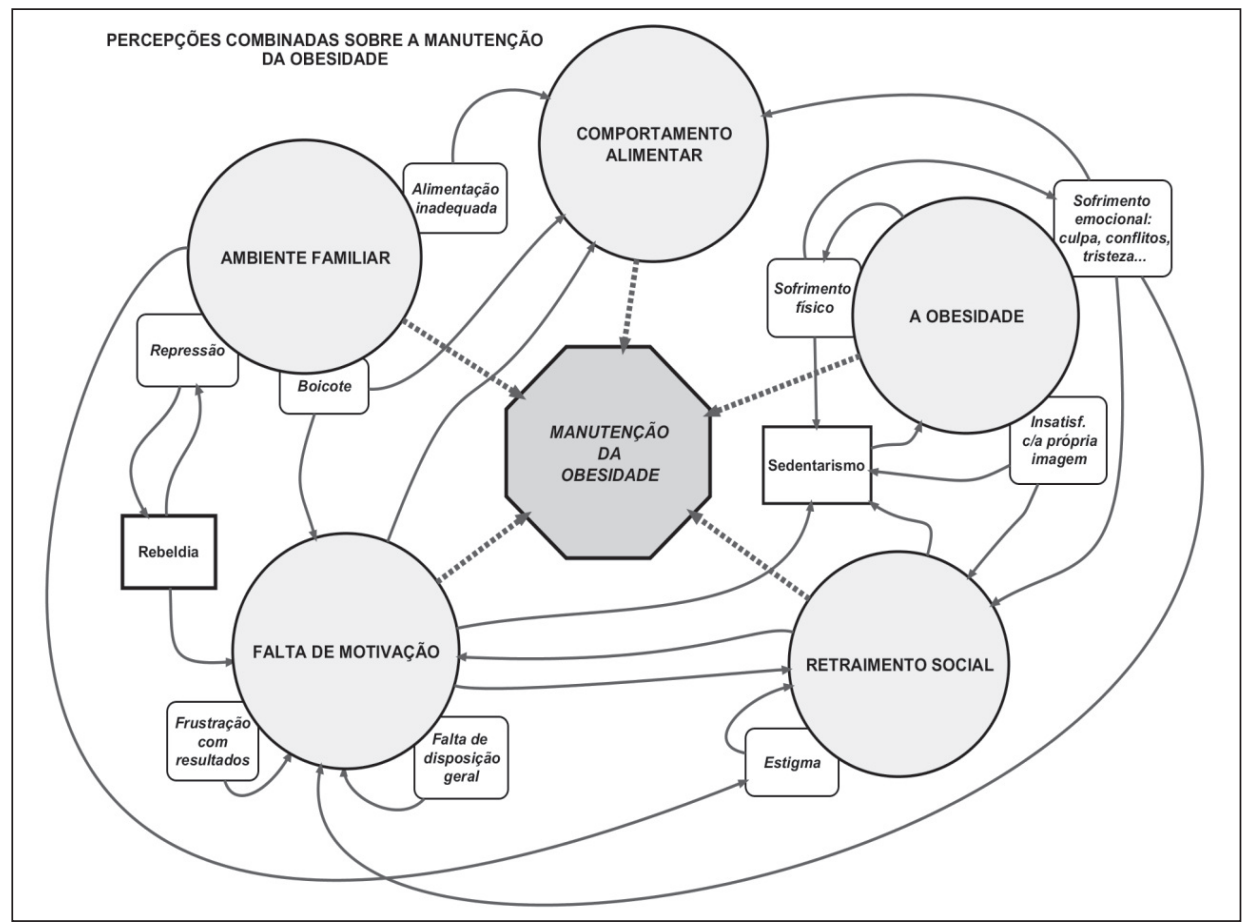

\section{Considerações finais}

Nos obesos deprimidos estudados neste trabalho, a percepção da própria obesidade, manifestada também pelo sofrimento físico, pelo sofrimento emocional e pela insatisfação com a autoimagem, implica sua própria manutenção. $\mathrm{O}$ sofrimento físico, referido como crônico e incapacitante, promove dificuldades no cuidado consigo mesmo e nas atividades físicas, levando ao sedentarismo, também relacionado à insatisfação com a autoimagem, ao estigma e à descriminação.

Os sujeitos da pesquisa percebem seu consumo alimentar como inadequado; entretanto, este é referido como associado a fatores psicossociais, ou seja, eles os apontam e desejam mudar a situação. Mas existem desânimo, falta de motivação e desistência. Isso nos permite inferir que a abordagem nutricional 
isolada pode não ser suficiente para mobilizar as mudanças necessárias para o tratamento e controle da obesidade.

Logo, para um atendimento integral ao obeso com depressão na atenção primária, se faz necessário também compreender, identificar e tratar os aspectos psicodinâmicos individuais, o que implica uma abordagem multidisciplinar, com associação entre métodos, os quais incluem, além da orientação nutricional e atividades físicas, intervenções psicoterápicas direcionadas para o tratamento dos sofrimentos psíquicos ligados à obesidade. Atualmente, as psicoterapias mais recomendadas são: psicodinâmica, interpessoal, psicoeducacional e cognitivo-comportamental, em grupo ou com famílias (GALVÃO; PINHEIRO; NUNES, 2006).

É necessário também diagnosticar e tratar, quando presentes, os transtornos mentais associados, sobretudo a depressão (SEGAL; CARDEAL; CORDÁS, 2002). No entanto, quando o uso de medicamentos for indicado, é importante lembrar que vários antidepressivos estão implicados no ganho de peso, podendo contribuir para a baixa adesão ao tratamento da obesidade (DEMÉTRIO; SOARES, 2008). Nesse contexto, percebe-se a importância da integração de especialistas de diversas áreas, incluindo os de saúde mental, com uma abordagem que considere os diversos fatores relacionados ao cuidado da obesidade, para ampliar a resolutividade de atendimento a essa população, o que compreende também a necessidade de capacitação dos profissionais de saúde.

Enfim, ao indicar que as percepçôes dos obesos deprimidos sobre sua obesidade se organizam numa teia complexa de fatores psicossociais mutuamente reforçadores, esperamos que esta pesquisa possa oferecer subsídios para a elaboração de estratégias de controle e enfrentamento da obesidade, buscando a integralidade, com acompanhamento multidisciplinar e intervenções diferenciadas para esta população.

Entre outras estratégias, podem ser consideradas as técnicas de grupos, por sua efetividade e baixo custo (WERUTSKY; BARROS, 2000), e também o envolvimento das famílias (BURD, 2004).

O trabalho com grupos tem como objetivo alcançar a construção coletiva do conhecimento, podendo ser um polo aglutinador dos profissionais e usuários, principalmente quando é realizado por equipe multidisciplinar. [...] O papel do profissional não é somente repassar informações, mas sim estimular a problematização, o saber pensar criticamente, fazendo com que o usuário se torne sujeito da ação, ou seja, um ser autônomo, com seu próprio conhecimento (BRASIL, 2006, p.54-55). 
O trabalho em grupo pode ter uma proposta educativa, com participação de

vários profissionais, onde cada um desenvolve um tema sob forma de palestra, ou serem baseados na troca, na construção, no compartilhar do conhecimento entre os profissionais, os usuários e a comunidade (BRASIL, 2006). Na atenção primária, são utilizados três modelos básicos de grupo: de "sala de espera", dirigidos aos usuários que aguardam a consulta, "fechados" (com participantes e tempo delimitados) e "abertos" (BRASIL, 2006).

O trabalho com grupos de famílias pode ter o mesmo objetivo e abordagens. Sua importância decorre do fato de que os hábitos alimentares se constroem e reforçam no ambiente familiar. Como exemplo de técnica de grupo, a cognitivocomportamental tem como características principais: o estabelecimento de metas, o automonitoramento, o controle de estímulos, a solução de problemas, a reestruturação cognitiva e a prevenção de recaídas (BERKEL, 2005).

Em resumo, é como sujeito, e apenas dessa forma, que o obeso com depressão pode começar a efetivamente lidar com seu problema. ${ }^{1}$

\section{Referências}

AMERICAN PSYCHIATRIC ASSOCIATION. DSM IV- TR: Manual diagnóstico e estatístico de transtornos mentais. 4a ed. rev. Porto Alegre: Artmed, 2002.

ALVARENGA, M. A mudança na alimentação e no corpo ao longo do tempo. In: PHILIPPI, S.; ALVARENGA, M. (Org.). Transtornos alimentares: uma visão nutricional. São Paulo: Manole, 2004. p. 1-19.

ARAÚJO, B. Aspectos psicológicos da alimentação. In: PHILIPPI, S.; ALVARENGA, M. (Org.). Transtornos alimentares: uma visão nutricional. São Paulo: Manole, 2004. p. 104-117. ASSOCIAÇÃO BRASILEIRA PARA ESTUDO DA OBESIDADE E DA SÍNDROME METABÓLICA. Obesidade e depressão: Associação Recíproca, São Paulo, 2009. Disponível em: <http://www.abeso.org.br/noticia/458/obesidade>. Acesso em: 20 jan. 2011.

. Doenças Desencadeadas ou agravadas pela obesidade, São Paulo, 2011. Disponível em: <http://www.abeso.org.br/artigos/obesidade>. Acesso em: 20 maio 2011.

BALL, K.; ATLANTIS, E. Association between weight perception and psychological distress. International Journal of Obesity, v.32, p.715-721, abr. 2008. Disponível em: <http:// www.ncbi.nlm.nih.gov/pubmed/18408736>. Acesso em: 10 set. 2012.

BARBOSA, S. Subjetividade e complexidade social: contribuições ao estudo da depressão. Physis: Rev. Saúde Coletiva, Rio de Janeiro, v. 16, n. 2, p. 317-350, 2006.

BARDIN, L. Análise de conteúdo. Lisboa: Ediçōes 70, 2004. 287 p. 
BENEDETTI, C. Funcionamento familiar e cirurgia da obesidade. In: FRANQUES, A.; LOLI, M. Contribuiçôes da psicologia na cirurgia da obesidade. São Paulo: Vetor, 2006. p. 105-113.

BENEDETTO, C. Questões investigativas sobre obesidade e gênero. In: FRANQUES, A.; LOLI, M. Contribuiçôes da psicologia na cirurgia da obesidade. São Paulo: Vetor, 2006. p. 115-123.

BERKEL, L.A. et al. Behavioral interventions for obesity. American Diet Association, v. 105, p. 35-43, May 2005.

BRASIL. Ministério da Saúde. Secretaria de Atenção à Saúde. Departamento de Atenção Básica. Obesidade. Brasília, 2006. 108p. (Caderno de Atenção Básica, 12).

. Ministério da Saúde. Portal da Saúde 2012. Disponível em: http://portal.saude. gov.br/saude/visualizar_texto.cfm? Acesso em: 7 out 2011.

- Ministério da Saúde. Conselho Nacional de Saúde. Comissão Nacional de Ética em Pesquisa. Resolução no 196 /1996. Brasília, 1996.

BRAZ, M. A construção da subjetividade masculina e seu impacto sobre a saúde do homem: reflexão bioética sobre justiça distributiva. Ciência \& Saúde Coletiva, Rio de Janeiro, v. 10, n. 1, p. 94-104, 2005.

BRUCE, B. Does depression cause obesity? A meta-analysis of longitudinal studies of depression and weight control. Journal of Health Psychology, v. 13, n. 8, p. 1190-1197, 2008. BURD, M. Obesidade e família. In: MELlO FILHO, J.; BURD, M. (Orgs.). Doença e família. São Paulo: Casa do Psicólogo, 2004. p. 299-321.

BUSSE, S. Anorexia, bulimia e obesidade. São Paulo: Manole, 2007. 366 p.

CAMPOS, G.W.; BARROS, R.B.; CASTRO, A.M. Avaliação da política nacional de promoção da saúde. Ciência \& Saúde Coletiva, Rio de Janeiro, v. 9, n. 3, p. 746, 2004.

CASH, T.F.; DEAGLE, E.A. The nature and extent of body image disturbances in anorexia nervosa and bulimia: meta-analisys. Intern Journal of Eating Disorder, v. 22, p. 107-25, set. 1997.

CHALUB, M. O homem não aceita mais ficar triste. Associação Brasileira de Psiquiatria, 2010. Disponível em: http://abpbrasil.org.br. Acesso em: 18 jul. 2012.

COUTINHO, J.; GENTIL, P.C.; TORAL, N. A. Desnutrição e obesidade no Brasil: o enfrentamento com base na agenda única da nutrição. Cad. Saúde Pública, Rio de Janeiro, v. 24, supl. 2, p. 332-334, 2008.

CUNHA, J.A. Manual da versão em português das Escalas Beck. São Paulo: Casa do Psicólogo, 2001.

DÂMASO, A. Obesidade. Rio de Janeiro: Medsi, 2003. 590 p. 
DEMÉTRIO, F.N.; SOARES, O.T. Depressão relacionada a outras enfermidades. Rio de Janeiro: Elsevier, 2008. 103p.

DIXON, J.B.; DIXON, M.E.; O'BRIEN, P. E. Depression in association with severe obesity: chances with weight loss. Arch Intern Medical, v. 163, n. 17, p. 2058-65, 2003.

DOBROW, J.; KAMENETZ, C.; DEVLIN, J. Aspectos psiquiátricos da obesidade. Rev. Bras. Psiquiatria. São Paulo, v. 24, n. III, p. 63-67, 2002.

FELIPPE, F.M. O peso social da obesidade. 2001, 282p. Tese (Doutorado em Serviço Social) - Pontifícia Universidade Católica do Rio Grande do Sul, Rio Grande do Sul, 2001.

FIGUEIREDO, W. Assistência à saúde dos homens: um desafio para os serviços de atenção primária. Ciência \& Saúde Coletiva. Rio de Janeiro, v. 10, n. 1, p. 105-9, 2005.

FLECK, M. Temas atuais em depressão. Rev Bras Psiquiatr. São Paulo, v. 31, n. 1, maio 2009. Disponível em: <http://www.scielo.br/scielo.php> Acesso em: 7 out. 2011.

FORTES, S. O paciente com dor. In: BOTEGA, N. Prática psiquiátrica no hospital geral: interconsulta e emergência. Porto Alegre: Artmed, 2002. p. 339- 351.

FREITAS, F.L. Tristeza e depressão. 2011. 66p. Dissertação (Mestrado em Saúde Coletiva) Universidade Federal de Santa Catarina, Centro de Ciências da Saúde, Florianópolis, 2011.

GALVÃO, A.; PINHEIRO, A.; NUNES, M. Psicoterapia dos transtornos alimentares. In: NUNES, A. et al. (Orgs.). Transtornos alimentares e obesidade. Porto Alegre: Artmed, 2006, p.137.

GOFFMAN, E. Estigma: notas sobre a manipulação da identidade deteriorada. Rio de Janeiro: Livros Técnicos e Científicos, 1988. 160p.

GREJANIN, D.K. et al. As percepções sobre o "ser obeso" sob a ótica do paciente e dos profissionais de saúde. Rev. bras. crescimento desenvolv. Hum. São Paulo, v. 17, n. 3, p. $104-$ 32, 2007. Disponível em: <http://www.revistasusp.sibi.usp.br/pdf/rbcdh/v17n3/04.pdf>. Acesso em: 8 jul. 2012.

HAMID, T.K.A. Thinking in circles about obesity. Monterey: Springer, 2009467 p.

INSTITUTO BRASILEIRO DE GEOGRAFIA E ESTATÍSTICA. Pesquisa de Orçamentos Familiares (POF) 2008-2009. Análise da Disponibilidade Domiciliar de Alimentos e Estado Nutricional no Brasil. Rio de Janeiro: 2010. Disponível em: <http://www.ibge.gov. br/home/estatistica/populacao/condicaodevida/pof/2008_2009_aval_nutricional/default. shtm> Acesso em: 20 jun. 2011

KAPLAN, H.; SADOCK, B. Compêndio de psiquiatria. Porto Alegre: Artmed, 2007. 1584 p. MALTA, D.C. et al. A construção da vigilância e prevenção das doenças crônicas não transmissíveis no contexto do Sistema Único de Saúde. Rev.Epidemiologia e Serviços de Saúde. Brasília, v. 15, n. 3, set. 2006. 
MARCHESINI, S. Aspectos psicológicos da obesidade mórbida, 2007 Disponível em: < http:// www.symbolon.com.br/artigos>. Acesso em: 10 jun. 2011.

MATOS, M.I. Quando o hábito alimentar se transforma em transtorno alimentar. In: FRANQUES, A.; LOLI, M. Contribuiçôes da psicologia na cirurgia da obesidade. São Paulo: Vetor, 2006. p. 137-159.

MATTOS, R.A.; LUZ, M. Sobrevivendo ao estigma da gordura: um estudo socioantropológico sobre obesidade. Rio de Janeiro: Revista de Saúde Coletiva, v. 19, n. 2, p. 489-507, 2009.

MINAYO, M.C. de S. O desafio do conhecimento: pesquisa qualitativa em saúde. 8 a ed. São Paulo: Hucitec, 2004. 406p.

MORAES, A. A percepção de pacientes obesos com depressão sobre os fatores envolvidos na manutenção da obesidade: uma investigação no Programa Saúde da Família - Lapa. Rio de Janeiro, 2011. 98p. Dissertação (Mestrado em Saúde da Família), Universidade Estácio de Sá, Rio de Janeiro, 2011.

OLIVEIRA, V.; LINARDI, R.; AZEVEDO, A. Cirurgia bariátrica: aspectos psicológicos e psiquiátricos. Rev Psiquiatria Clinica. São Paulo, v. 31, n. 4, p. 199-201, abr. 2004.

ONYIKE, C.U. et al. Is obesity associated with major depression? Results from the third national heallth and nutrition examination survey. Am Jour of Epidemiol, v. 15, p. 11391147, dez. 2003.

PINHEIRO, A.; FREITAS, S.; CORSO, A. Uma abordagem epidemiológica da obesidade. Rev Nutr Campinas, v. 17, n. 4, p. 523-533, out/dez. 2004.

PINTO, M.; BOSI, M.L. Muito mais do que pe(n)sam: percepçôes e experiências acerca da obesidade entre usuárias da rede pública de saúde de um município do Nordeste do Brasil. Physis Revista de Saúde Coletiva. Rio de Janeiro, v. 20, n. 2, p. 443-457, 2010.

SANTOS, L.; RABINOVICH, E. Situações familiares na obesidade exógena infantil do filho único. Saude soc., São Paulo, v. 20, n. 2, abr/ jun 2011.

SEGAL, A.; CARDEAL, M.V.; CORDÁS, T.A. Aspectos psicossociais e psiquiátricos da obesidade. Rev. Psiquiatria Clínica, São Paulo, v. 29, n. 2, p. 81-89, 2002.

SIMON, G. et al. Association between obesity and depression in middle-aged women. Gen Hosp Psychiatry, v. 30, n. 1, 2008.

SOARES, O. Depressão e obesidade. In: DEMÉTRIO, F.N.; SOARES, O.T. (Orgs.). Depressão relacionada a outras enfermidades. Rio de Janeiro: Elsevier, 2008.

STAFFIERE, J.R. A study of social stereotype of body image in children. J.Pers. Soc.Psychol., n. 7, p. 101-104, 1967. 
STUNKARD, A.; WADDEN, T. Psychological aspects of severe obesity. Am. Soc. for

Clin. Nutr., v. 55, n. 2, p. 5245-5325, 1992. Disponível em: <http://ajcn.nutrition.org/ content/55/2/524S > Acesso em: 8 out. 2011.

TARDIDO, A; FALCÃO, M. O impacto da modernização na transição nutricional e obesidade. Rev Bras Nutr Clinica, v. 21, n. 2, p. 117-24, 2006.

UEHARA, M.; MARIOSA, L. Obesidade: etiologia e história natural. In: CLAUDINO, A.; ZANELLA, M. (Orgs.). Guias de Medicina Ambulatorial e Hospitalar Unifesp/ Escola Paulista de Medicina. São Paulo: Manole, 2005. p. 195-201.

WERUTSKY, C.A.; BARROS, T.M. Grupo com obesos. In: MELLO FILHO e cols. Grupo e corpo. Porto Alegre: Artmed, 2000. p 285-293.

WORLD HEALTH ORGANIZATION. Obesity: preventing and managing the global epidemic: report of a WHO consultation on Obesity. Geneva, 1998 Disponível em: . Acesso em: 20 jun. 2011.

. Conquering depression, 2011. Disponível em: http://www.searo.who.int/ Acesso em: 20 jun. 2011.

10 facts on obesity. Geneva, 2012. Disponível em: <http://www.who.int/features/ factfiles/obesity/facts/en/index1.html> Acesso em: 6 set.2012.

\section{Nota}

${ }^{1}$ A.L. Moraes realizou a pesquisa de campo, a busca bibliográfica e elaborou o artigo. E.C. Almeida e L.B. Souza colaboraram na redação e na revisão do artigo. 


\section{Perceptions of depressed obese people about the} factors associated with the maintenance of their obesity: an investigation in a Family Health Program in Rio de Janeiro city

According to WHO, obesity and depression are among the greatest Public Health problems worldwide. Some studies show important association between these diseases that can worsen the prognostic for co-morbidities, reduce compliance with treatment, increase morbidity and mortality, and deteriorate the functional and psychosocial roles of obese people. This paper comes out from a Master thesis research in Family Health, aiming to investigate the perception of depressed obese people about the factors linked with the maintenance of their obesity. This is a qualitative exploratory study in a Family Health Service in Rio de Janeiro city. Sixty-eight obese people were randomly selected, and, among them, 21 showed depression scores, measured by the Beck Depression Inventory. Out of these, 19 were interviewed and the resulting data were analyzed through the content analysis technique. Some of the main categories found were physical suffering, sedentary behavior, emotional suffering, self-image dissatisfaction, inadequate eating behavior, family and social relationships, and the motivation for change. They were grouped in five thematic axes: the perception of one's obesity, the feed behavior, the family environment, the social retraction, and the lack of motivation. Taken together, the perceptions of these people were mapped in a complex net of mutually reinforcing factors associated with the maintenance of the obesity, and this is aggravated by depression. These results could help to conceive new strategies for an integral and specific care of obese people, mostly those who are depressed.

> Key words: obesity; depression; self-concept; feeding behavior; Family Health Program. 